\title{
Laser interstitial thermal therapy for subependymal giant cell astrocytoma: technical case report
}

\author{
David Y. A. Dadey, PhD, ${ }^{1,2}$ Ashwin A. Kamath, MD, ${ }^{1}$ Eric C. Leuthardt, MD, ${ }^{1}$ and \\ Matthew D. Smyth, MD'

\begin{abstract}
1Department of Neurological Surgery and 2Medical Scientist Training Program, Washington University School of Medicine, St. Louis, Missouri
\end{abstract}

\begin{abstract}
Subependymal giant cell astrocytoma (SEGA) is a rare tumor occurring almost exclusively in patients with tuberous sclerosis complex. Although open resection remains the standard therapy, complication rates remain high. To minimize morbidity, less invasive approaches, such as endoscope-assisted resection, radiosurgery, and chemotherapy with mTOR pathway inhibitors, are also used to treat these lesions. Laser interstitial thermal therapy (LITT) is a relatively new modality that is increasingly used to treat a variety of intracranial lesions. In this report, the authors describe two pediatric cases of SEGA that were treated with LITT. In both patients the lesion responded well to this treatment modality, with tumor shrinkage observed on follow-up MRI. These cases highlight the potential of LITT to serve as a viable minimally invasive therapeutic approach to the management of SEGAs in the pediatric population.
\end{abstract}

http://thejns.org/doi/abs/10.3171/2016.7.FOCUS16231

KEY WORDS laser interstitial thermal therapy; LITT; SEGA; subependymal giant cell astrocytoma; ventriculostomy

$\mathrm{T}$ uBEROus sclerosis complex (TSC) is a disorder caused by genetic mutations in TSCl or TSC2.,3 These mutations are autosomal dominant and result in the growth of hamartomas in various tissues, including the brain. ${ }^{3}$ Among the hamartomas seen in TSC are subependymal giant cell astrocytomas (SEGAs). SEGAs arise almost exclusively in patients with TSC. ${ }^{1}$ Up to $20 \%$ of pediatric TSC cases present with SEGA, and while SEGAs are low-grade tumors (WHO Grade I), complications can arise due to their propensity to obstruct the flow of CSF within the brain. ${ }^{16}$ The tendency for SEGAs to occur in periventricular tissues often leads to blockage of the foramen of Monro. ${ }^{4,8}$ This can result in life-threatening hydrocephalus and sequelae including seizures and focal neurological deficits. ${ }^{4,18}$ Currently, standard treatment for these tumors involves resection. ${ }^{2}$ However, a study of patients who underwent surgery for SEGAs between 2000 and 2009 revealed a postoperative complication rate of $48.9 \% .{ }^{17}$ The majority of these were procedure-related complications, which may have been associated with the extent of dissection required to access the deep location of SEGAs. This highlights a need for alternative, minimally invasive surgical strategies - a need that remains largely unmet due to the difficulty posed by the deep location of these tumors. ${ }^{2,17}$ Laser interstitial thermal therapy (LITT) is a relatively new method of targeting such deep-seated lesions and has been successfully used for thermal ablation of tumors in several organ systems including the brain. $6,11,12,14$ There are a limited number of reports that discuss the application of LITT to SEGAs.$^{15}$ Hence, in this report, we describe two cases of SEGA that were treated with LITT at our institution.

\section{Case Reports \\ Case 1}

History and Examination

This patient was a 13-year-old girl initially diagnosed with TSC in utero during prenatal ultrasound, when she was found to have cardiac rhabdomyomas. Since birth, she has had an extensive history of epilepsy, autism, and developmental delay, along with classic cutaneous lesions, hamartomas, and SEGAs associated with TSC. She underwent two craniotomies for resection of bilateral SEGAs

ABBREVIATIONS LITT = laser interstitial thermal therapy; PICU = pediatric intensive care unit; SEGA = subependymal giant cell astrocytoma; TDT = thermal damage threshold; TSC $=$ tuberous sclerosis complex. 
at 4 years of age, and fenestration of the septum pellucidum during her first craniotomy to reduce future risk of hydrocephalus. Her recovery from these procedures was uneventful and she continued to be followed with serial imaging over the years. In 2015, a new enlarging mass was observed at her prior resection bed in her left lateral ventricle. Her parents also noted new behavioral changesnamely, abandoning the family during outings and arrests in her ambulation. Her neurological examination was normal except for left ocular esotropia.

\section{Initial Imaging}

MRI showed postsurgical changes consistent with previous bilateral frontal horn SEGA resections. Multiple T2 signal hyperintense foci were seen in the cortical and subcortical matter, which were consistent with tubers of TSC. A $1.7 \times 1.4 \times 2.1-\mathrm{cm}$ lobular enhancing mass was present in the left lateral ventricle, which had increased in size from 6 months previously, when it measured $1.1 \times 1 \times 1.2$ cm (Fig. 1).

\section{Operation}

As the mass was likely to be a recurrent SEGA, her family was again counseled and offered various treatment options. Conservative management, medical management with everolimus (Novartis), a third craniotomy, and the option of MRI-guided LITT were discussed. Ultimately the family elected LITT. On the day of surgery, the StealthStation MRI-guided Navigation System (Medtronic) was coregistered with her preoperative MRI scans to allow for trajectory planning. A right frontal approach with a trajectory crossing through the right lateral ventricle to reach the contralateral left lateral ventricle was chosen to allow for probe insertion into the core of the tumor. A stab incision was made to allow for drilling of a $4.5-\mathrm{mm}$ bur hole and for placement of a 4.5-mm-outerand 3.2-mm-inner-diameter anchor bolt. Then, a biopsy needle was used to cannulate the left ventricle and create an entry point on the tumor surface. Using frameless stereotactic guidance, a Monteris Diffusing Tip Laser Probe was advanced into the tumor. Once inserted to the desired depth, intraoperative 1.5-T MRI (IMRIS Inc.) was used to track thermal ablation with real-time MR thermometry. Using thermal damage threshold (TDT) lines, the conformality of the treatment zone was monitored to ensure complete coverage of the SEGA. The TDT yellow and blue lines are lines that indicate the region of tissue heated to the thermal dose equivalent of $43^{\circ} \mathrm{C}$ for 2 minutes and $43^{\circ} \mathrm{C}$ for 10 minutes, respectively (Fig. 2). After completion of ablation, intraoperative MRI revealed expansion of the tumor consistent with ablation-induced swelling. We therefore performed additional ablation to expand the treatment zone to encompass the new tumor margins. Upon completion of this additional ablation, the laser probe and bolt were removed, and the scalp was closed with Monocryl 3-0 suture.

\section{Postoperative Course}

After surgery, the patient was monitored overnight in the pediatric intensive care unit (PICU). She did well and was discharged the following day. She was seen for fol-
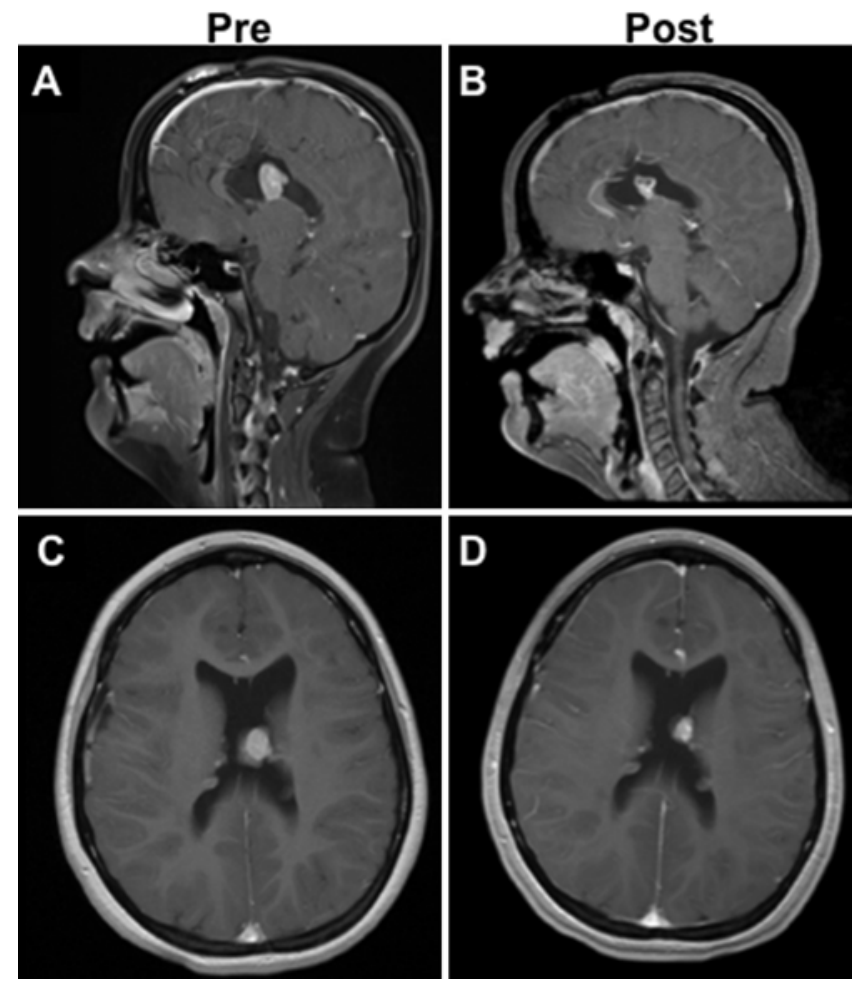

FIG. 1. Case 1. Preablation sagittal (A) and axial (C) Gd-enhanced T1weighted MR images showing the SEGA. Postablation sagittal (B) and axial (D) Gd-enhanced T1-weighted MR images showing treatment effects 3 months after surgery.

low-up 3 months later, and her parents indicated she had made significant improvement in her behaviors and exhibited no complications since surgery. On neurological examination her status was normal except for stable left ocular esotropia. MRI performed at the time showed interval decrease in the size of the SEGA, which measured $1.2 \times 0.9 \times 1.2 \mathrm{~cm}$. After 9 months of ongoing observation since her procedure, she continues to do well.

\section{Case 2}

History and Examination

This 14-year-old girl had a history of TSC. She was diagnosed at the age of 2 years when she developed infantile spasms and refractory epilepsy, and she was found to have a spontaneous TSC mutation on genetic evaluation. Her seizures have been well controlled since she began taking topiramate, and though she does have the classic tubers and a SEGA, her disease course overall has been relatively benign. On routine neuroimaging, an interval enlargement of her SEGA was observed, along with dilation of her lateral ventricles. Despite these findings, she did not indicate experiencing any headaches and had a normal neurological status on examination.

\section{Initial Imaging}

MRI showed multiple subcortical T2 signal hyperintense foci consistent with hamartomas of TSC. In addition, a large enhancing mass was observed in her left lateral ventricle near the foramen of Monro, most likely 


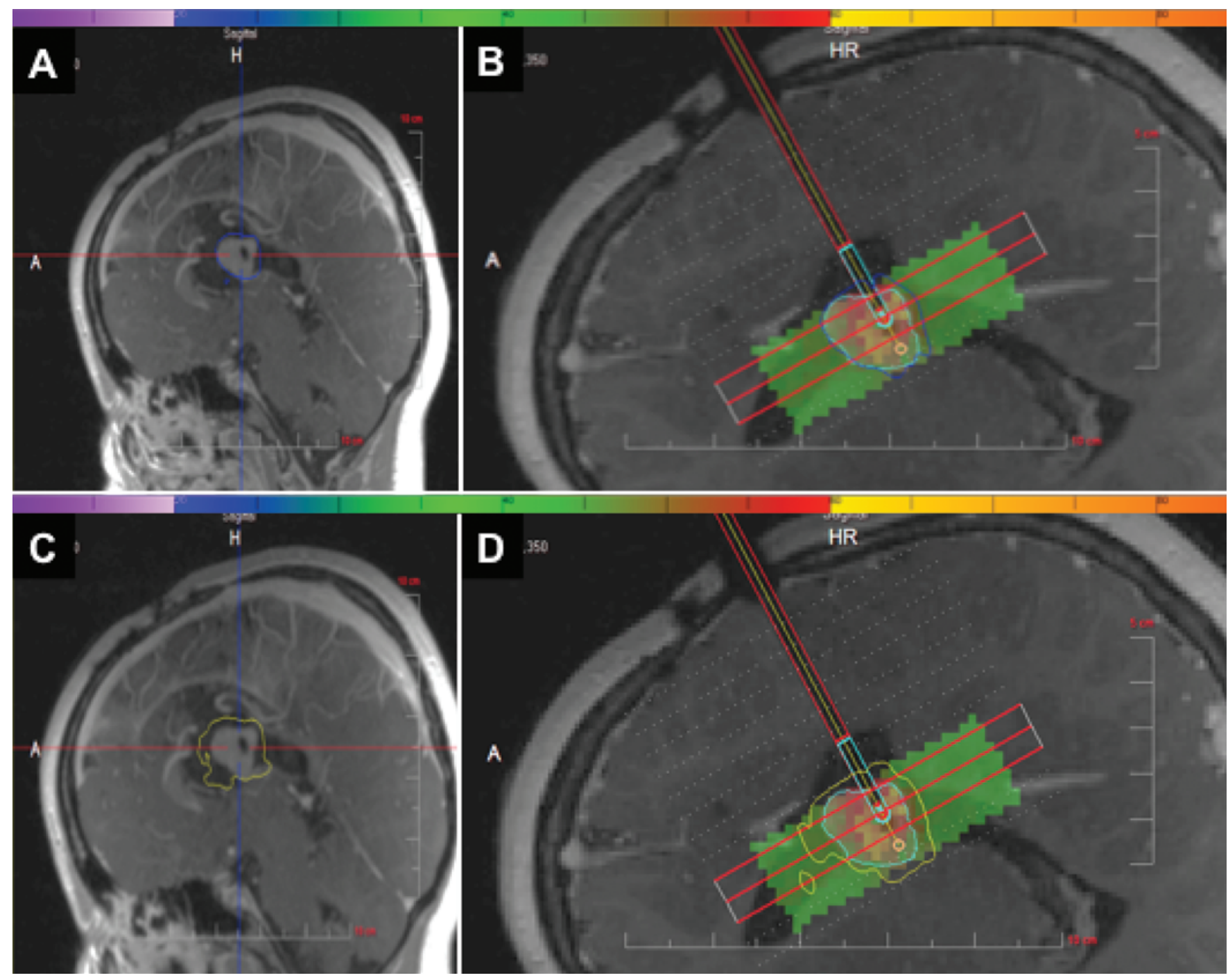

FIG. 2. Case 1. Laser interstitial thermal therapy. Intraoperative Gd-enhanced T1-weighted MR images showing the probe tip within the tumor along with the planned treatment zone (blue circle in $\mathbf{A}$ ) and the TDT line (yellow circle in C). In panels B and D, the red lines perpendicular to the probe represent slices through the treatment zone that were used to monitor thermal ablation in real time.

representing a SEGA. The mass measured $0.7 \times 1.0 \times 1.1$ $\mathrm{cm}$ on imaging 6 months previously, and it was found to have grown to a size of $1.6 \times 1.7 \times 1.9 \mathrm{~cm}$. Interval bilateral enlargement of the lateral ventricles suggestive of obstructive hydrocephalus was also noted (Fig. 3).

\section{Operation}

Out of concern for the marked increase in her tumor burden and radiographic evidence of hydrocephalus, we recommended therapeutic intervention. Multiple options including LITT, craniotomy, radiosurgery, and medical management were discussed with the family, but they were most interested in LITT as a definitive, minimally invasive surgical option. Due to the location of the tumor proximal to the foramen of Monro and the possibility of postablation tumor swelling, we planned for the addition of a ventriculostomy during surgery. In addition, we felt she would be a good candidate for treatment with the ROSA robotic device-assisted LITT (Medtech). ROSA is a robotic stereotactic navigation platform with an arm capable of motion with 6 degrees of freedom, allowing for accurate frameless stereotaxy. We secured the patient's bed frame to the ROSA robot and proceeded to co-register the machine with the patient's cranial landmarks and preoperative scans. A trajectory using a frontal approach to reach the left lateral ventricle was chosen based on preoperative identification of a pathway avoiding cortical blood vessels. We targeted the exact entry point with ROSA assistance. We made a stab incision at the entry point and drilled a 4-mm bur hole through which a Medtronic biopsy needle was inserted and used to obtain tumor tissue samples. Then, an anchor bolt was placed within the bur hole, after which ROSA was used to determine the distance between the bolt edge and the tumor. This distance was used to determine the insertion depth of the laser probe required to reach the base of the tumor. ROSA was then removed to allow for placement of the laser probe. Intraoperative MRI was used to confirm that the probe was within the lesion and was subsequently used to monitor thermal ablation. LITT was delivered to a treatment zone spanning $90 \%$ 

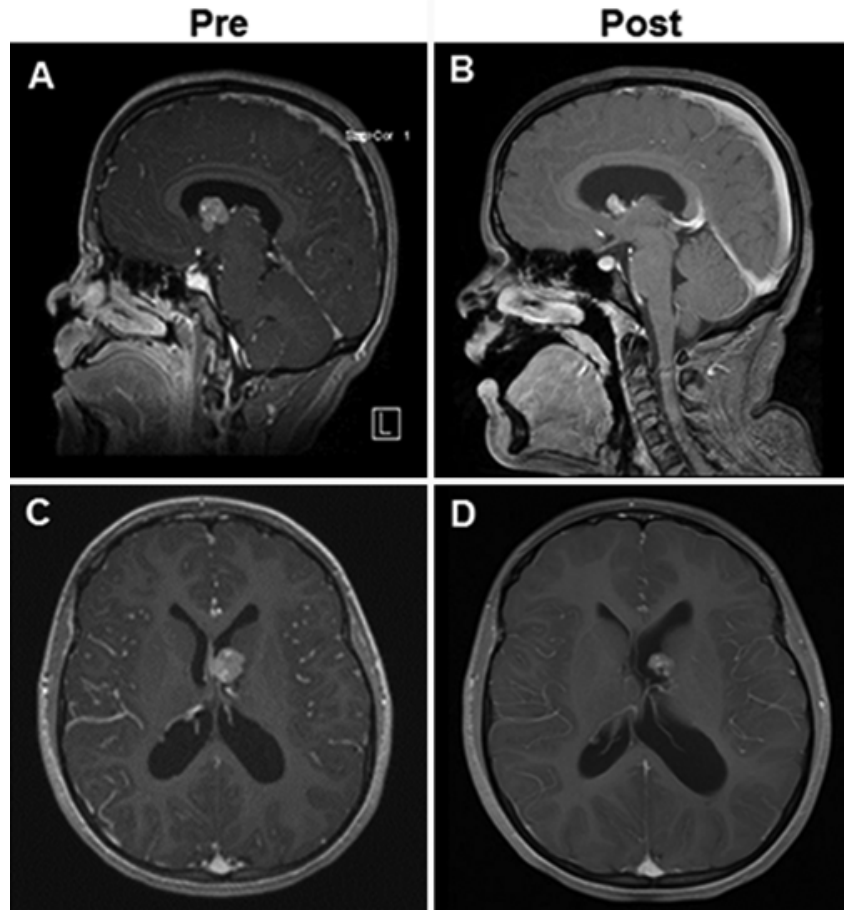

FIG. 3. Case 2. Preablation sagittal (A) and axial (C) T1-weighted MR images with Gd contrast showing the SEGA. Postablation sagittal (B) and axial (D) T1-weighted MR images with Gd contrast showing treatment effects 4 months after surgery.

of the tumor, as the eccentricity of the probe placement relative to the lesion did not allow for complete ablation without risk of damage to adjacent normal tissues. Upon completion of the procedure, we removed the probe and bolt and inserted and anchored a ventricular catheter prior to closure of the incision as previously performed.

\section{Postoperative Course}

The patient was admitted to the PICU after surgery, where she was monitored overnight. Over subsequent days, she did quite well and the ventriculostomy drain was eventually clamped. However, she developed progressive headaches and experienced two episodes of CSF leakage at the ventriculostomy incision site on the scalp. CT scanning revealed worsened ventriculomegaly. The drain was opened, after which her intracranial pressure normalized and her headaches resolved. Drainage continued for another 72 hours, and on a second clamp trial, repeat CT showed stable ventricular size, and the drain was successfully removed. The patient was discharged 9 days after surgery. Analysis of her biopsy samples confirmed the diagnosis of SEGA. At 4-month follow-up, she had remained symptom free and her parents noted general improvements in her behavior and personality. Her neurological status remained grossly normal. MRI performed at this time showed shrinkage of the SEGA and overall stable ventricular size with slightly increased left ventriculomegaly.

\section{Discussion}

SEGAs are slow-growing tumors that tend to develop within the first 2 decades of life. ${ }^{7}$ Thus, the current man- agement of these tumors involves frequent surveillance for developing hydrocephalus and changes in tumor growth rate. ${ }^{16}$ Surgery remains the standard therapy and is often considered curative if gross-total resection is achieved. However, the outcomes of surgery can vary, as one study found that excision of SEGAs greater than $3 \mathrm{~cm}$ in diameter was associated with $67 \%$ risk for surgery-related complications. ${ }^{10}$ In that study, adverse events after surgery were found to correlate with tumor burden and patient age, with patients younger than 3 years having greater risk. ${ }^{10}$ Furthermore, incomplete excision may be associated with tumor recurrence, necessitating repeat resection. ${ }^{16,17}$ In an effort to avoid the drawbacks of radical surgery, medical therapies for SEGAs have been a subject of significant investigation. Specifically, mTOR inhibitors have been shown to have efficacy in clinical trials, where tumor volume reductions of greater than $50 \%$ were observed in patients treated with everolimus., 5 Though these results are promising, additional investigation is required to determine if long-term therapy is required to prevent SEGA regrowth. Another noninvasive strategy is Gamma Knife radiosurgery, but there is limited evidence to support its efficacy as a treatment for SEGA. ${ }^{13}$

To our knowledge, there are very few studies investigating LITT as a treatment for SEGAs. One review article mentions an unpublished series that included SEGAs among a variety of pediatric brain lesions treated with LITT. ${ }^{15}$ However, the outcomes were not specifically described. We therefore, herein, illustrate two cases representing our experience with LITT as a therapy for SEGAs. In both cases, LITT was tolerated well by the patients, and there were no intraoperative complications. In both cases, we observed tumor shrinkage on follow-up neuroimaging, suggesting that LITT may have therapeutic efficacy in treatment of SEGAs. Both families indicated improved symptomatology after laser ablation, and for the patient in Case 1 the family also reported reduced seizure frequency and improved overall quality of life. Seizure frequency in the patient in Case 2 did not change. This is likely due to the multifactorial nature of TSC and differences in the underlying pathological basis of their seizures. Patient 1 , at 9 months after laser ablation, continues to remain free of any postoperative complications. Patient 2, at 4 months since her procedure, is also doing well despite a somewhat prolonged postoperative hospital course due to transient obstructive hydrocephalus. We postulate that this progression was due to postablation tumor swelling and that the resulting obstruction was more pronounced because the SEGA was closer to the foramen of Monro.

Transient peritumoral edema is a known treatment effect of LITT in various tumor types. ${ }^{6}$ In cases of SEGA, anticipating the downstream effects of this edema may be of higher importance given that a large proportion of these tumors are close to the foramen of Monro. Furthermore, patients may already present with hydrocephalus at the time of surgery, and it is possible that LITT may lead to transient worsening of their CSF flow obstruction. Our experience with Case 2 suggests that preemptive ventriculostomy could be beneficial when performing ablation of SEGAs. By providing a safety valve in case of aggravated hydrocephalus, ventriculostomy preserves the option of 
immediate pressure relief without necessitating repeat surgical intervention in the immediate postoperative period. Additionally, ventriculostomy is well suited to the transient nature of postablation tumor edema, as it allows for gradual weaning and may obviate the need for a more permanent shunt. The patient in Case 1, due to her prior septostomy and known patency of the foramen of Monro contralateral to her SEGA, was deemed to have a low risk of post-LITT hydrocephalus. Ventriculostomy was therefore not performed in her case.

This report is limited by its retrospective design and limited number of cases and, as such, is not amenable to meaningful statistical analysis. Also, the use of two different methods for stereotactic guidance may confound the overall efficacy observed, as the initial placement of the laser probe can impact the extent of treatment delivery. Lastly, longer follow-up is needed to comment on the long-term effect of LITT on SEGA growth.

\section{Conclusions}

The development of minimally invasive techniques for treating SEGAs is needed to avoid morbidity associated with open resection. At our institution, we have found that LITT can be used to treat SEGAs. In both patients described in this report, tumor ablation was achieved without intraoperative complication and resulted in improved symptomatology. Given the proximity of SEGAs to the foramen of Monro and the possibility of transient postablation tumor edema, we devised a method in which ventriculostomy was performed after the LITT procedure. This allowed for effective management of transiently worsened hydrocephalus during the postoperative period (Case 2). Though larger studies are needed with longer follow-up to evaluate overall treatment efficacy, our experience suggests that ventriculostomy should be considered in patients receiving LITT as a treatment for SEGA.

\section{References}

1. Beaumont TL, Godzik J, Dahiya S, Smyth MD: Subependymal giant cell astrocytoma in the absence of tuberous sclerosis complex: case report. J Neurosurg Pediatr 16:134-137, 2015

2. Beaumont TL, Limbrick DD, Smyth MD: Advances in the management of subependymal giant cell astrocytoma. Childs Nerv Syst 28:963-968, 2012

3. Crino PB, Nathanson KL, Henske EP: The tuberous sclerosis complex. N Engl J Med 355:1345-1356, 2006

4. Cuccia V, Zuccaro G, Sosa F, Monges J, Lubienieky F, Taratuto AL: Subependymal giant cell astrocytoma in children with tuberous sclerosis. Childs Nerv Syst 19:232-243, 2003

5. Franz DN, Agricola K, Mays M, Tudor C, Care MM, Holland-Bouley K, et al: Everolimus for subependymal giant cell astrocytoma: 5-year final analysis. Ann Neurol 78:929-938, 2015

6. Hawasli AH, Bagade S, Shimony JS, Miller-Thomas M, Leuthardt EC: Magnetic resonance imaging-guided focused laser interstitial thermal therapy for intracranial lesions: singleinstitution series. Neurosurgery 73:1007-1017, 2013

7. Jóźwiak S, Nabbout R, Curatolo P: Management of subepen- dymal giant cell astrocytoma (SEGA) associated with tuberous sclerosis complex (TSC): Clinical recommendations.

Eur J Paediatr Neurol 17:348-352, 2013

8. Jung TY, Kim YH, Jung S, Baek HJ, Lee KH: The clinical characteristics of subependymal giant cell astrocytoma: five cases. Brain Tumor Res Treat 3:44-47, 2015

9. Komotar RJ, Starke RM, Connolly ES, Sisti MB: mTOR inhibitors in the treatment of subependymal giant-cell astrocytomas associated with tuberous sclerosis. Neurosurgery 68:N24-N25, 2011

10. Kotulska K, Borkowska J, Roszkowski M, Mandera M, Daszkiewicz P, Drabik K, et al: Surgical treatment of subependymal giant cell astrocytoma in tuberous sclerosis complex patients. Pediatr Neurol 50:307-312, 2014

11. Missios S, Bekelis K, Barnett GH: Renaissance of laser interstitial thermal ablation. Neurosurg Focus 38(3):E13, 2015

12. Mohammadi AM, Schroeder JL: Laser interstitial thermal therapy in treatment of brain tumors - the NeuroBlate System. Expert Rev Med Devices 11:109-119, 2014

13. Park KJ, Kano H, Kondziolka D, Niranjan A, Flickinger JC, Lunsford LD: Gamma Knife surgery for subependymal giant cell astrocytomas. Clinical article. J Neurosurg 114:808813,2011

14. Rahmathulla G, Recinos PF, Kamian K, Mohammadi AM, Ahluwalia MS, Barnett GH: MRI-guided laser interstitial thermal therapy in neuro-oncology: a review of its current clinical applications. Oncology 87:67-82, 2014

15. Riordan M, Tovar-Spinoza Z: Laser induced thermal therapy (LITT) for pediatric brain tumors: case-based review. Transl Pediatr 3:229-235, 2014

16. Roth J, Roach ES, Bartels U, Jóźwiak S, Koenig MK, Weiner HL, et al: Subependymal giant cell astrocytoma: diagnosis, screening, and treatment. Recommendations from the International Tuberous Sclerosis Complex Consensus Conference 2012. Pediatr Neurol 49:439-444, 2013

17. Sun P, Kohrman M, Liu J, Guo A, Rogerio J, Krueger D: Outcomes of resecting subependymal giant cell astrocytoma (SEGA) among patients with SEGA-related tuberous sclerosis complex: a national claims database analysis. Curr Med Res Opin 28:657-663, 2012

18. Torres OA, Roach ES, Delgado MR, Sparagana SP, Sheffield E, Swift D, et al: Early diagnosis of subependymal giant cell astrocytoma in patients with tuberous sclerosis. J Child Neurol 13:173-177, 1998

\section{Disclosures}

Dr. Leuthardt reports having a consulting relationship with Monteris Medical and ownership in Neurolutions, OsteoVantage, and Pear Therapeutics.

\section{Author Contributions}

Conception and design: Smyth. Acquisition of data: Kamath, Leuthardt, Smyth. Analysis and interpretation of data: Dadey, Kamath, Smyth. Drafting the article: Dadey. Critically revising the article: all authors. Reviewed submitted version of manuscript: all authors. Approved the final version of the manuscript on behalf of all authors: Dadey. Administrative/technical/material support: Leuthardt, Smyth. Study supervision: Smyth.

\section{Correspondence}

David Y. A. Dadey, Department of Neurological Surgery, Washington University in St. Louis, 660 S Euclid Ave., Box 8057, St. Louis, MO 63110. email: ddadey@wustl.edu. 